\title{
Meniscal ossicle
}

\author{
Seema Rohilla $\cdot$ Rohtas K. Yadav $\cdot$ Raj Singh \\ Ashish Devgan · Dhara B. Dhaulakhandi
}

Received: 31 October 2008/Accepted: 22 June 2009/Published online: 10 July 2009

(C) Springer-Verlag 2009

\begin{abstract}
Meniscal ossicle, or bone within the substance of meniscus, is a rare entity and commonly confused with a loose body both clinically and radiologically. MRI is the modality that can definitely diagnose meniscal ossicle and avoid unnecessary diagnostic arthroscopy. Here we report one such case diagnosed using MRI; this patient is doing well without surgery one year after diagnosis.
\end{abstract}

Keywords Meniscal ossicle $\cdot$ MRI ·

Conservative treatment

\section{Introduction}

Meniscal ossicle is mature lamellar and cancellous bone containing fatty bone marrow surrounded by hyaline cartilage within the substance of menicus. It is a rare entity and must be differentiated from various other entities like chondrocalcinosis, avulsion at the site of attachment of posterior cruciate ligament or the posterior horn of the meniscus, and avulsions of the semimembranosus tendon,

S. Rohilla $\cdot$ R. K. Yadav

Department of Radiodiagnosis and Imaging,

Pt. B.D. Sharma Post Graduate Institute of Medical Sciences,

University of Health Sciences, Rohtak 124001, Haryana, India

R. Singh · A. Devgan

Department of Orthopedics, Pt. B.D. Sharma Post Graduate Institute of Medical Sciences, University of Health Sciences, Rohtak 124001, Haryana, India

D. B. Dhaulakhandi $(\square)$

Department of Biotechnology and Molecular Medicine,

Pt. B.D. Sharma Post Graduate Institute of Medical Sciences, University of Health Sciences, Rohtak 124001, Haryana, India e-mail: BTMM.submissions@gmail.com as well as from a loose body, as it requires different management. MR is the modality of choice for conclusively diagnosing meniscal ossicles and thus avoiding unnecessary intervention, both diagnostic and therapeutic. We share our experience with one such symptomatic case of meniscal ossicle.

\section{Case report}

A 25-year-old male farmer presented with right knee pain and swelling for six months. The pain was intermittent in nature. The patient also had intermittent locking of the knee joint. There was no recent history of trauma or any other relevant past history. Clinical examination showed mild swelling without any restriction of movement. The patient was thought to have internal derangement of knee, and so an MRI of the knee was done on a $1.5 \mathrm{~T}$ scanner (Philips Intera, Germany) using a dedicated knee coil. T1W, T2W and proton density weighted (PDW) sequences with and without fat suppression were used to image the knee in the sagittal and coronal planes. A well-defined lesion was identified in the substance of the posterior horn of the medial meniscus, isointense to bone marrow on all pulse sequences, and suppression of the signal on PDW fat suppressed images, with a complete hypointense rim (see Figs. 1 and 2). This represented a meniscal ossicle. There was no other associated finding in the knee. The articular cartilage was also normal. The patient was treated conservatively with analgesics, anti-inflammatory drugs and activity modification (restricting rigorous sports activities and discouraging a cross-legged sitting position). His symptoms subsequently disappeared and he is well after one year of follow-up. The patient provided his consent to the publication of this case report. 
Fig. 1 a Coronal proton density weighted image of the knee showing a small lesion (arrow), isointense to marrow in the posterior horn of the medial meniscus, with a hypointense rim. b Coronal PDW fat suppressed image confirms the isointensity of the lesion to the marrow
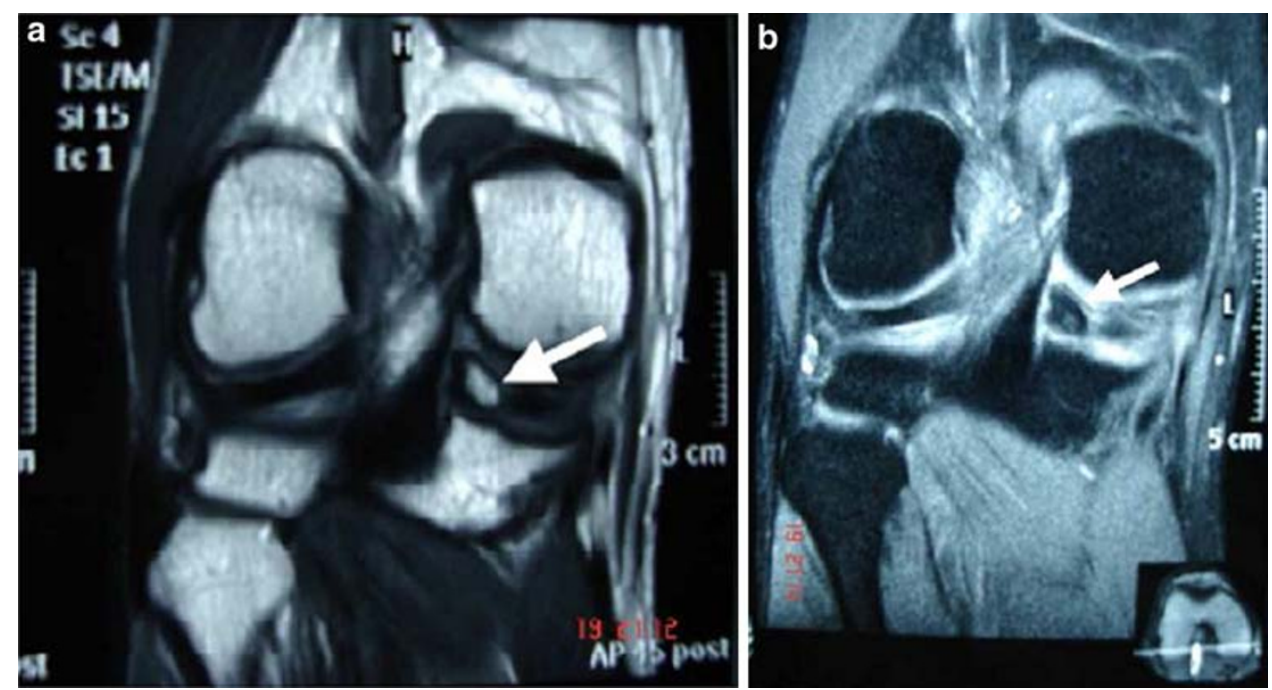

Fig. 2 a Sagittal gradient echo images showing that the lesion (arrow) is isointense to the bone marrow and has a rim of hyaline cartilage. b Sagittal T1W image showing that the lesion is isointense to marrow with a hypointense rim
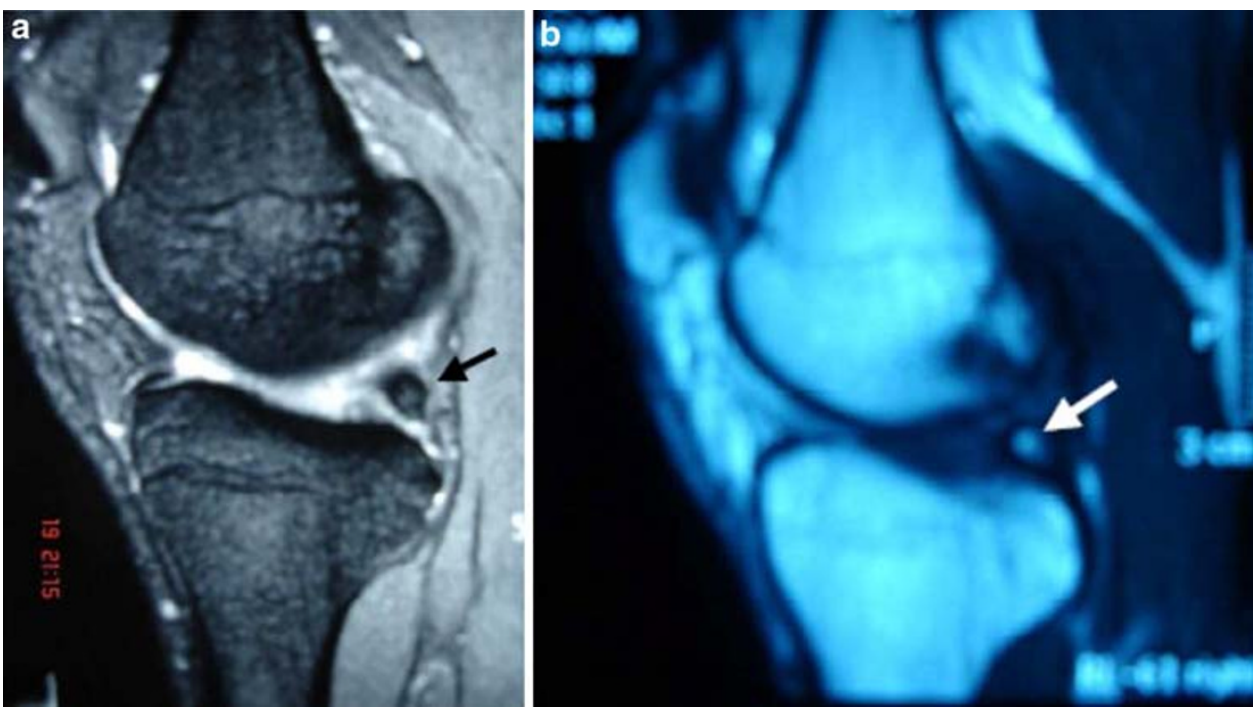

\section{Discussion}

Meniscal ossicle was first reported in 1931 and later by Burrows [1] and by Watson-Jones and Roberts [2] in 1934. To the best of our knowledge, it has been reported 42 times so far $[3,4]$. Most of the reported cases were located in the posterior horn of the medial meniscus $[5,6]$. The meniscal ossicle in our case was also located in the posterior horn of the medial meniscus.

Ossicles are mature lamellar and cancellous bone containing fatty bone marrow surrounded by hyaline cartilage [7]. Many theories have been put forward regarding the etiology of meniscal ossicles. They may be vestigial structures, as they are common in rodents, domestic cats and Bengal tigers [2], or they may represent a degenerative phenomenon due to the ossification of mucoid degeneration [5]; however, this is unlikely, as ossicles occur mostly in younger men, before the onset of significant mucoid degeneration [8]. A traumatic etiology has also been put forth, suggesting that the ossicles represent heterotopic ossification [9]; or they may represent bone fragments arising from the tibial attachment of the meniscal root insertion. This theory is supported by the fact that the most common location for meniscal ossicles is in the posterior horn of the medial meniscus [1,8], which shows a strong attachment to the tibia and reduced mobility and is thus more prone to an avulsion tear. The normal contour of the adjoining bone on MRI, however, argues against this theory [8]. In short, there is no definite consensus on the etiology of meniscal ossicles.

Most patients complain of intermittent pain, as in our case. According to Van Breuseghem et al. [3], a locking sensation is usually not experienced as expected with a free intra-articular body, but this is not always true, as our patient had intermittent locking, and the two patients reported by Schnarkowski et al. [8] also had locking. So 
this clinical sign is not a definite criterion for distinguishing a meniscal ossicle from a loose body.

Radiologically, the commonest misdiagnosis is a loose body. A lateral X-ray knee shows a triangular radiodense opacity projected over the posterior joint line. On fluoroscopy, the ossicle moves with the tibia during knee rotation [7]. USG can distinguish between loose bodies and ossicles, but it is operator dependent. This differentiation can also be made with arthrography and CT arthrography, but these are invasive procedures [10].

MRI can easily depict the location of ossicles within the substance of the meniscus [8,11], thus distinguishing them from loose bodies, chondrocalcinosis, osteochondritis dessicans and semimembranosus and popliteal tendon avulsions [8]. Their characteristic isointensity to adjacent normal bone marrow, along with a hypointense rim, further distinguishes them from loose bodies and chondrocalcinosis, the latter being hypointense on T1W images. MRI also helps to identify associated abnormalities like meniscal tears, ligament tears and avulsions, cartilage damage, and synovial effusion, and hence is the modality of choice for assessing such cases.

Meniscal ossicles must be distinguished from loose bodies, as loose bodies require surgical removal while meniscal ossicles can be managed conservatively (as in our case), and arthroscopic removal can be retained as a last option. Van Breuseghem et al. [3] and Glass et al. [12] recommend surgical removal of the loose body, and further add that meniscal ossicles can be managed conservatively. The literature is silent about the appropriate type of conservative management. Arthroscopy is a definitive modality, and arthroscopic removal of ossicles is usually the last resort [4]. MRI can definitely distinguish between the two, and diagnostic arthroscopy can be avoided.

Conflict of interest statement The authors declare that they have no conflict of interest related to the publication of this manuscript.

\section{References}

1. Burrows HJ (1934) Two cases of ossification in the internal semilunar cartilage. Br J Surg 21:404-410

2. Watson-Jones R, Roberts RE (1934) Calcification, decalcification and ossification. Br J Surg 21:461-499

3. Van Breuseghem I, Geusens E, Pans S, Brys P (2003) The meniscal ossicle revisited. JBR-BTR 86:276-277

4. Lawande MA, Tavri S, Patkar DP, Pungavkar SA, Narang J (2008) Meniscal ossical. Indian J Radiol Imaging 18(2):128-129

5. Yu JS, Resnick D (1994) Meniscal ossicle: MR imaging appearance in three patients. Skeletal Radiol 23(8):637-639

6. Conforty B, Lotem M (1993) Ossicles in human menisci: report of two cases. Clin Orthop 293:225-228

7. Prabhudesai V, Richards PJ (2003) Radiological appearance as a meniscal ossicle develops: a case report and review of literature. Injury 34:378-382

8. Schnarkowski P, Tirman PF, Fuchigami KD, Crues JV, Butler MC, Genant HK (1995) Meniscal ossicale: radiographic and MR imaging findings. Radiology 196:47-50

9. Berg EE (1991) The meniscal ossicle: the consequence of meniscal avulsion. Arthroscopy 7:241-243

10. Martinoli C, Bianchi S, Spadola L, Garcia J (2000) Multimodality imaging assessment of meniscal ossical. Skeletal Radiol 29:481484

11. Tuite MJ, De Smet AA, Swan S, Kneene JS (1995) MR imaging of a meniscal ossicle. Skeletal Radiol 24:543-545

12. Glass RS, Barnes WM, Kells DU, Thomas S, Campbell C (1975) Ossicles of knee menisci: report of seven cases. Clin Orthop Relat Res 111:163-171 